\title{
Space, science and the bottom line
}

In April, planetary scientist Alan Stern joined NASA as associate administrator for science, putting him in charge of the agency's $\$ 5.5$-billion science budget. Now he must juggle more than 90 space missions and 3,000 grants with focuses ranging from Earth to the distant Universe.

You've announced extensions to a number of missions lately. Does this mean you're cutting future missions? Not at all - it's an effective way to get more science done with our budget. Often this gives you more bang for the buck by providing important new science for very little additional cost. With these extensions, we're going to get two new comet visits and a search for extrasolar planets for about $15 \%$ of the cost that either of the Stardust and Deep Impact missions took to build. And there will be more future missions.

\section{What about costs?}

Cost can be held down by properly scoping missions to begin with and then closely managing to that budget. There's been too much management by open chequebook, and that will not be continued. We are changing the culture about overruns and we are not going to tolerate them as was done in the past. The Kepler mission [to find Earthlike planets] is one example. When they came saying they needed more money I said 'no' repeatedly. And they found a way to stay within budget.

The Mars programme is the biggest planetary programme at NASA, but what's the status of a mission to bring back rocks?

Mars sample return has been on the to-do list for 35 years, but it's had a very frustrating life cycle. It has repeatedly become very popular and then grown to be so large as to be unaffordable. But because it's been so highly ranked by the scientific community, I want to see us finally do it. So I've asked Doug McCuistion, our Mars programme director, to draw up architectures for an affordable Mars sample-return mission, and to design it to cost. To remain affordable it will have to be a Chevy sample-return instead of a Cadillac version, but I know what we've got from overly ambitious, Cadillac-like Mars sample-return mission plans: zero grams of martian rock.

\section{Will it really} happen this time? To show people I'm serious about getting started, I've asked the spacecraft builders at the Jet Propulsion Laboratory to add a sample-caching ability to the our next rover, the Mars Science Laboratory [planned to launch in 2009]. As that drives around it will pick up samples and store them for possible pick-up by the sample-return mission a decade hence. This will give us critical practice at sampling on Mars, and it will also give us the option of skipping a future rover to cache samples - meaning we can reduce the cost and shorten the time until a documented sample is back on Earth.

\section{Some scientists question the Vision} for Space Exploration, NASA's push to return astronauts to the Moon. What real science is there to be learned from such a programme?

Let's remember that Apollo was very important scientifically. It taught us that the Earth-Moon system was born in a giant impact, it taught us about the importance of magma oceans, and its samples may even have recorded the signature of the clearing of the Kuiper belt [a phase in the early Solar System when mass was lost from the asteroid belt]. With twenty-first-century technology we will be able to accomplish a lot more science when we return to the Moon than Apollo ever could with 1960s technology. The vision for space exploration is critically important for the country, and it's also extremely exciting for science.

Imagine: we'll be sending sophisticated 